\title{
Menstrual Cup-Associated Toxic Shock Syndrome
}

\author{
Christian Neumann, Rene Kaiser, Judith Bauer \\ Joint Medical Service (Germany), Department of Internal Medicine, Military Hospital Ulm, Ulm, Germany
}

Received: $18 / 06 / 2020$

Accepted: $24 / 06 / 2020$

Published: $22 / 07 / 2020$

How to cite this article: Neumann C, Kaiser R, Bauer J. Menstrual cup-associated toxic shock syndrome. EJCRIM 2020;7: doi:10.12890/2020_001825.

Conflicts of Interests: The Authors declare that there are no competing interests.

Acknowledgements: Radiographic images used with kind approval of the Radiologic Department (Military Hospital Ulm).

This article is licensed under a Commons Attribution Non-Commercial 4.0 License

\section{ABSTRACT}

Toxic shock syndrome (TSS) is a rare inflammatory response syndrome associated with an infection by toxigenic strains of Staphylococcus aureus or group A $\beta$-haemolytic Streptococcus. We report a rare case of menstrual TSS associated with usage of a menstrual cup. The diagnosis was established through case definition criteria and supported by vaginal cultural growth of Staphylococcus aureus with evidence of TSS toxin 1 (TSST-1). The patient received prophylactic intravaginal clindamycin in an individual approach to reduce the risk of recurrence. No relapse was reported in the 12 months following discharge.

\section{LEARNING POINTS}

- TSS should be considered in female patients presenting with fever, rash, hypotension and current menses. Prompt initiation of antibiotics and supportive care is critical.

- Menstrual cups may be a rare cause of TSS.

- Prophylactic antibiotic therapy may reduce the risk of relapse.

\section{KEYWORDS}

Menstrual cup, menstrual toxic shock syndrome

\section{CASE DESCRIPTION}

We report the case of a 33-year-old woman presenting to the emergency department with acute onset of fever, rash and hypotension. Her initial vital signs were remarkable for a low blood pressure of $80 / 54 \mathrm{mmHg}, 39^{\circ} \mathrm{C}$ body surface temperature, heart rate of 134 bpm, respiratory rate of 25 breaths per minute and oxygen saturation of $95 \%$ while breathing ambient air.

On physical examination, an altered mental status, a diffuse erythematous macular rash on her trunk and aphthous ulcerations in the oral cavity were noted.

Laboratory assessments on admission revealed elevated inflammatory markers, acute kidney injury and elevated lactate levels.

On further evaluation of the medical history at the emergency department, the patient reported having unusual persistent vaginal burning and pain during the last 3 days of menstruation, which began 4 days before presentation. She was using a menstrual cup, which was changed, boiled and disinfected twice a day during usage. The patient had been using this very same cup for the previous 4 years.

Having obtained blood cultures, the patient was started on a broad-spectrum antibiotic (piperacillin and tazobactam) and intravenous fluids. Despite adequate fluid therapy, additional vasopressors were necessary because of persistent hypotension in the medical intensive care unit. 
Bedside echocardiography and abdominal ultrasound did not show any evidence of cardiac vegetations or acute intra-abdominal pathology. The left ventricular ejection fraction was normal. A chest $x$-ray in the supine position revealed reticular nodular opacities (Fig. 1). A vaginal examination revealed no foreign bodies but was painful. An additional vaginal swab for culture purposes was obtained during that examination. In reviewing the recent medical history, the physical examination and the laboratory findings, we suspected menstrual toxic shock syndrome (mTSS) and added clindamycin to suppress toxin synthesis (Table 1).

The clinical course was complicated by ARDS with correlating CT findings (Fig. 2) and cardiogenic shock, confirmed by a highly reduced ejection fraction and global hypokinesia as observed with bedside echocardiography.

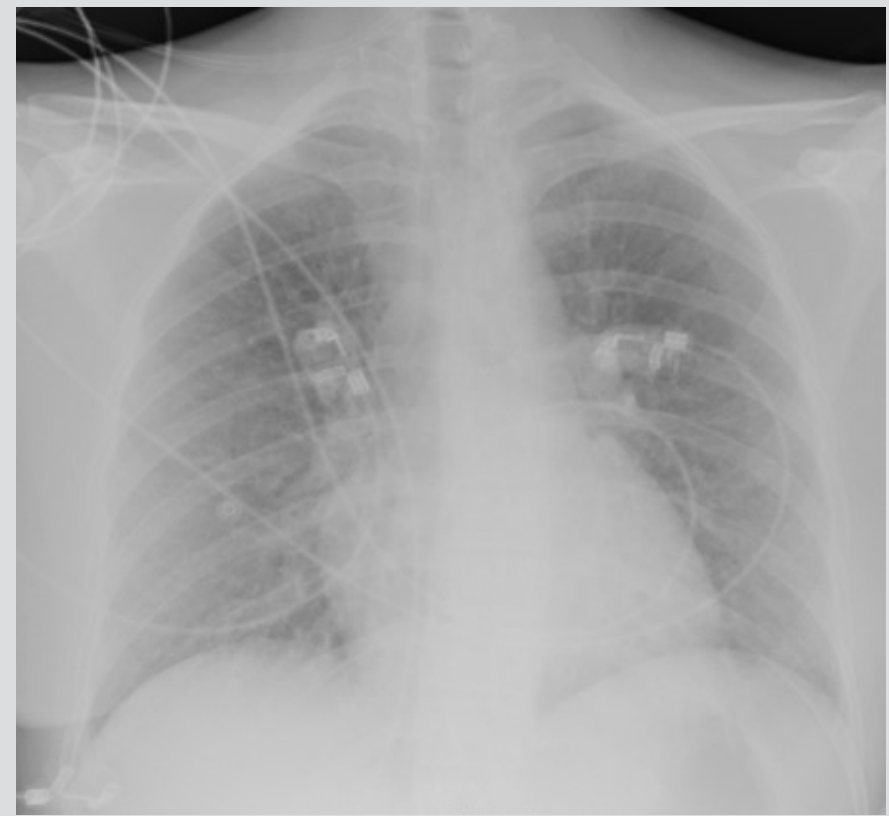

Figure 1. X-ray of the chest on Day 1 of hospitalization in the supine position showing bilateral pulmonary reticular nodular opacities

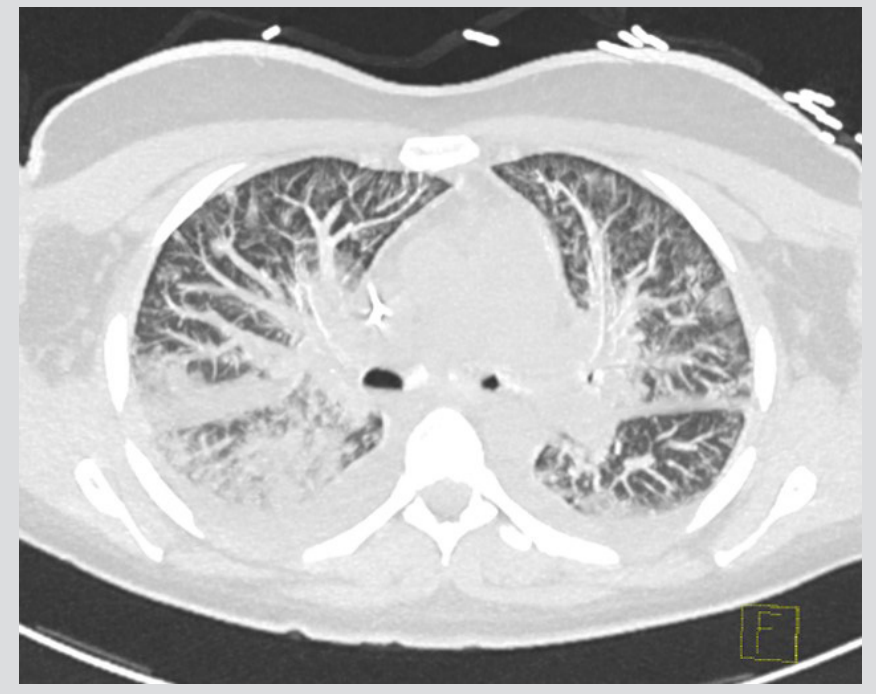

Figure 2. Computed tomography of the chest showing bilateral opacities consistent with acute respiratory distress syndrome on Day 2 of hospitalization

\section{CDC Case Definition 2011}

\section{Clinical Criteria}

- Fever: temperature greater than or equal to $102 . F^{\circ}$ (greater than or equal than $38,9^{\circ}$ )

- Rash: diffuse macular erythrodema

- Desquamation: 1-2 weeks after onset of rash

- Hypotension: systolic blood pressure less than or equal to $90 \mathrm{~mm} \mathrm{Hg}$ for adults or less that fifth percentile by age for children aged less than 16 years old

- Multisystem involvement (three or more of the following organ systems):

- Gastrointestinal: vomiting or diarrhea at onset of illness

- Muscular: severe myalgia or creatine phosphokinase level at least twice the upper limit of normal

- Mucous membrane: vaginal, oropharyngeal, or conjunctival hyperemia

- Renal: blood urea nitrogen or creatinine at least twice the upper limit of normal for laboratory or urinary sediment with pyuria (greater than or equal to 5 leukocytes per high-power field) in the absence of urinary tract infection

- Hepatic: total bilirubin, alanine aminotransferase enzyme, or asparate aminotransferase enzyme levels at least twice the upper limit of normal laboratory

- Hematologic: platelets less than 100,000/mm

- Central nervous system: disorientation or alterations in consciousness without focal neurologic signs when fever and hypotension are absent

- Blood or cerebrospinal fluid cultures: blood culture may be positive for Staphylococcus aureus

- Negative serologies for Rocky Mountain spotted fever, leptospirosis, or measles

Table 1. The CDC case definition for TSS. Probable TSS meets the laboratory criteria and 4 of the 5 clinical criteria. Confirmed TSS meets the laboratory criteria and all 5 of the clinical criteria, unless the patient dies before desquamation occurs CDC: Centers for Disease Control and Prevention; TSS: toxic shock syndrome 
Over the following 9 days vasopressor therapy was tapered. The patient was extubated on the 10th day of hospitalization as desquamation of the soles and palms began (Fig. 3).

Despite negative vaginal swab and skin cultures after antibiotic treatment we opted for topical usage of intravaginal clindamycin for 14 days as an individual approach to reduce the risk of recurrence as the patient had been through a severe course of mTSS.

No further recurrence was reported in the following 12 months and a full recovery of systolic function was achieved.

Positive culture samples were sent to a reference laboratory, which revealed Staphylococcus aureus (MSSA, clonal complex 22, spa type t1368) with evidence of TSS toxin 1 (TSST-1) in the latex agglutination test.

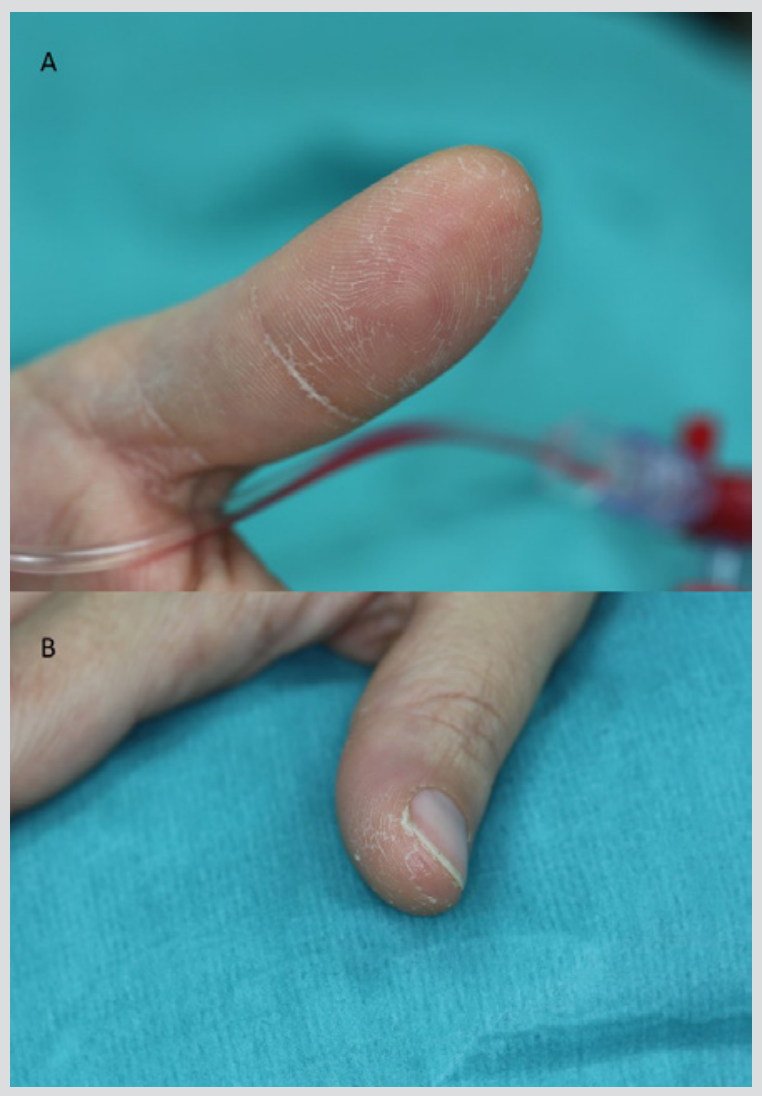

Figure 3. Beginning of desquamation on Day 10 after hospitalization for the left (A) and right (B) thumbs

\section{DISCUSSION}

Systemic infections with Staphylococcus aureus are associated with an increased mortality, particularly in toxin-induced systemic multi-organ failure such as with mTSS. The causative agent of staphylococcal-induced TSS is TSST-1, an exotoxin within the superantigen family. By bypassing antigen processing, superantigens can induce clonal T-cell proliferation resulting in massive cytokine release and organ damage ${ }^{[1]}$. Menstrual cups are made of silicon or rubber and are being increasingly used as a popular alternative to tampons. At the time of writing this report, menstrual cups have been reported only once in the literature as a cause of mTSS ${ }^{[2]}$.

The pathogenesis of mTSS associated with menstrual cups is still elusive and may be multifactorial in origin. Although the accumulation of blood may provide a medium for bacterial growth, an in vitro trail showed no detectable production of TSST-1 in menstrual cups ${ }^{[3]}$.

To reduce the risk of relapse of mTSS, discontinuation of tampon usage is recommended. However, relapse can occur in the absence of tampon usage and negative vaginal swab cultures ${ }^{[4,5]}$. Davis and colleagues observed recurrence of mTSS in 5 out of 30 women who discontinued tampon use during menses in a 5-month period after the initial episode ${ }^{[4]}$.

Despite the high recurrence rate, there are only a few general recommendations regarding antibiotic prophylaxis in women with mTSS. One possible prophylactic approach is the use of oral contraceptives $(\mathrm{OC})$, which may reduce the risk of recurrence by preventing menstruation ${ }^{[5]}$. Dixit and colleagues opted for oral rifampicin and clindamycin instead of OC in a 14-year-old girl who continued to experience recurrences 


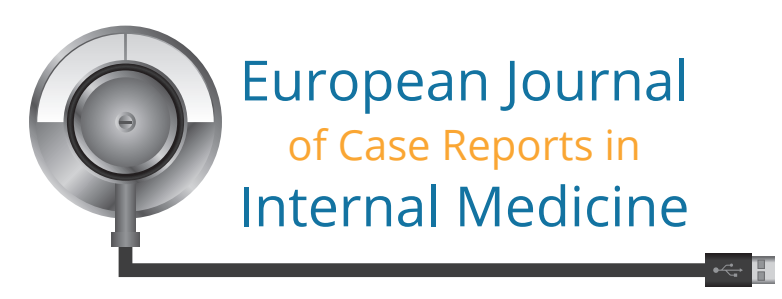

despite avoiding tampons. The rationale behind the antibiotic therapy was to clear a presumptive staphylococcal carrier state. The girl stayed free of recurrence ${ }^{[5]}$.

With respect to our patient, we opted for topical usage of intravaginal clindamycin as an individual approach to reduce the risk of relapse. In summary, it can be stated that mTSS is a rare disease and may be associated with menstrual cups. Antibiotic prophylaxis after mTSS may reduce recurrence but further research is still necessary.

\section{REFERENCES}

1. Low DE. Toxic shock syndrome: major advances in pathogenesis, but not treatment. Crit Care Clin 2013;29(3):651-675.

2. Mitchell MA, Bisch S, Arntfield S, Hosseini-Moghaddam SM. A confirmed case of toxic shock syndrome associated with the use of a menstrual cup. Can J Infect Dis Med Microbiol 2015;26(4):218-220.

3. Tierno PM, Hanna BA. Propensity of tampons and barrier contraceptives to amplify Staphylococcus aureus toxic shock syndrome toxin-I. Infect Dis Obstet Gynecol 1994;2(3):140-145.

4. Davis JP, Chesney PJ, Wand PJ, LaVenture M. Toxic-shock syndrome: epidemiologic features, recurrence, risk factors, and prevention. N Engl J Med 1980;303(25):1429-1435.

5. Dixit S, Fischer G, Wittekind C. Recurrent menstrual toxic shock syndrome despite discontinuation of tampon use: is menstrual toxic shock syndrome really caused by tampons? Australas J Dermatol 2013;54(4):283-286. 